\title{
Computation of a Three-Dimensional Flow in a Square Microchannel: A Comparison Between a Particle Method and a Finite Volume Method
}

\author{
D. Bento ${ }^{1,2}$, R. Lima ${ }^{2,3}$ and Joao M. Miranda ${ }^{2, *}$
}

${ }^{I}$ ESTiG, Polytechnic Institute of Bragança (IPB), Portugal. ${ }^{2}$ CEFT, Faculdade de Engenharia da Universidade do Porto (FEUP) Ruas Dr. Roberto Frias, 4200-465 Porto, Portugal. ${ }^{3}$ Mechanical Eng. Dep., University of Minho, Campus de Azurém, 4800-058 Guimarães, Portugal

\begin{abstract}
Traditional grid-based numerical methods, such as finite volume method (FVM), are not suitable to simulate multiphase biofluids (such as blood) at the microscale level. Alternatively, meshfree Lagrangian methods can deal with two or more finely dispersed phases moving relatively to each other. The Moving Particle Semi-Implicit Method (MPS), used in this study, is a deterministic particle method based on a Lagrangian technique to simulate incompressible flows. The advantages of particle methods over traditional grid-based numerical methods have motivated several researchers to implement them into a wide range of studies in computational biomicrofluidics. The main aim of this paper is to evaluate the accuracy of the MPS method by comparing it with numerical simulations performed by an FVM. Hence, simulations of a Newtonian fluid flowing through a constriction were performed for both methods. For the MPS, a section of the channel of $30 \times 11.5 \times 11.5 \mu \mathrm{m}$ was simulated using periodic boundary conditions. The obtained results have provided indications that, if the initial particle distance is sufficiently small, the MPS method can calculate accurately velocity profiles in the proposed channel.
\end{abstract}

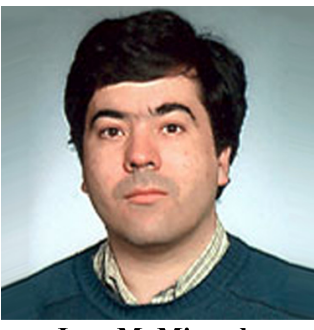

Keywords: Blood flow, finite volume method, moving particle semi-implicit method, navier-stokes equations.

\section{INTRODUCTION}

Blood is a physiological fluid that consists of plasma and different kinds of cells, such as red blood cells (RBCs), white blood cells (WBCs) and platelets [1]. Under healthy conditions human RBCs have a biconcave shape of approximately $8 \mu \mathrm{m}$ in diameter and are highly deformable, which allows them to pass through narrow capillaries with a diameter several times smaller than the RBC size [2].

From a macroscopic point of view the human blood can be regarded as a homogenous fluid. However, at the microscopic level the blood should be treated as a fluid mainly composed of different types of cells [1]. Consequently an excellent approach to investigate the microscopic mechanical behavior of blood flow is considering the human blood as a set of suspended discrete particles. Hence, particle methods [3] are a natural choice to perform blood flow simulations at a microscopic level.

Classical numerical techniques, such as finite element method (FEM), finite difference method (FDM) or finite volume method (FVM) are used to simulate blood flow at the macroscale level, since blood can be assumed as a homogeneous fluid.

Recently computer approaches using discrete particles have been proposed to investigate the dynamic behavior of blood cells in the blood flow, such as formation and destruction

\footnotetext{
*Address correspondence to this author at the Transport Phenomena Research Center (CEFT), Department of Chemical Engineering, Engineering Faculty University of Porto, Rua Dr. Roberto Frias, 4200-465 Porto, Portugal; Tel: +351 2250814 00; Fax: +351 2250814 40;

E-mail: jmiranda@fe.up.pt
}

of thrombi [4]. During the last decade several researchers $[1,3,5-7]$ have been developing a computer simulation method, known as Moving-Particle Semi-Implicit Method (MPS), to simulate blood flow at microscale level. By using this method they were able to analyze the RBC motion and deformation, platelets flow and their interactions in microvessels [1, 3, 5-7].

The use of particle methods for solving fluid dynamics problems is relatively recent. Although for grid based methods a large amount of knowledge has been accumulated over the years, in the case of particle methods the experience of the scientific community is more limited. The validation procedure of in house codes must be, therefore, more carefully investigated.

This paper presents a numerical study of a Newtonian fluid in order to identify constraints and ways to improve the MPS method and to investigate the accuracy of this method when compared with the existing traditional techniques. The results obtained by the MPS method were compared and validated with the results obtained by the FVM. This latter method was implemented in the commercial software Ansys Fluent. The flow of a Newtonian homogeneous fluid in a rectangular microchannel was selected as a benchmark since it can be accurately predicted by analytical and grid based methods.

\section{THEORY}

\subsection{Moving Particle Semi-Implicit Method (MPS)}

The Moving Particle Semi-implicit method (MPS) solves the incompressible Navier-Stokes equations by using a La- 
grangian particle approach. The original method was developed by Koshizuka and Oka in 1996 [8], and assuming incompressible viscous flow, the motion of all particles is determined using the MPS method. In the MPS method, particle motion is modeled by using the equation of continuity and Navier-Stokes equations with a semi-implicit timemarching algorithm [9]. The MPS method considers the Navier-Stokes equations as the conservation of mass and momentum in Lagrangian frame of reference:

$\frac{1}{\rho} \frac{D \rho}{D t}+\nabla \cdot u=0$

$\frac{D u}{D t}=-\frac{1}{\rho} \nabla p+g+v \nabla^{2} u$

where $u$ is the particle velocity vector; $t$ is time, $g$ is gravitational acceleration vector, $v$ is a kinematic viscosity, $p$ is the particle pressure and $\rho$ is the fluid density. The equation (1) is written in the form of compressible flow. In the MPS method, incompressibility is enforced by way of setting $D \rho / D t=0$ at each particle and calculation step. In the MPS method the advection term is automatically calculated through the tracking of particle motion; hence, the numerical diffusion arising from the successive interpolation of the advection function in Eulerian grid-based methods is controlled without the need of a sophisticated algorithm [10].

The interaction between neighboring particles is given by the kernel function $w(r)$, where $r$ is the distance between two particles [8]. This function describes the effect of distance on the influence of particle quantities. The kernel function must decrease with the distance between two particles [9]. The function is given by:

$w(r)=\left\{\begin{array}{cl}\frac{r_{e}}{r}-1 & \Leftarrow 0<r<r_{e} \\ 0 & \Leftarrow r_{e}<r\end{array}\right.$

where $r_{e}$ is the finite radius where there are interactions between particles in the MPS method, $r$ is the distance between two particles, $r=\left|r_{j}-r_{i}\right|$ for some $j$ and $i$ particles [8]. Note that the kernel function is infinity at $r=0$. This is beneficial for numerical stability in the model of incompressibility $[8,10]$. The particle number density at position $i$ is given by:

$n_{i}=\sum_{j \neq i} w\left(\left|r_{j}-r_{i}\right|\right)$

When the number of particles in unit volume is denoted by $N_{i}$. The relation between $n_{i}$ and $N_{i}$ is given by the equation:

$N_{i}=\frac{n_{i}}{\int_{V} w(r) d V}$

where $V$ is the whole region, excluding a central part occupied by particle $i$. Considering that all particles have the same mass, $m$, the fluid density is proportional to the particle number density, $\rho_{i}$, and can calculated through the equation:

$\rho_{i}=m N_{i}=\frac{m n_{i}}{\int_{V} w(r) d V}$

Thus, the continuity equation is satisfied if the particle number density is constant. This constant value is denoted by $n^{0}$.
A gradient vector between two particles $i$ and $j$ possessing scalar quantities $\phi_{i}$ and $\phi_{j}$ at the coordinates $r_{i}$ and $r_{j}$ is simply defined by $\left(\phi_{j}-\phi_{i}\right)\left(r_{j}-r_{i}\right) /\left|r_{j}-r_{i}\right|^{2}$. The gradient vector between particle $i$ and $j$ are weighted with the kernel function and averaged to obtain a gradient vector at particle $i$ :

$\nabla \phi_{i}=\frac{d}{n^{0}} \sum_{j \neq i}^{N}\left[\frac{\phi_{j}-\phi_{i}}{\left|r_{j}-r_{i}\right|^{2}}\left(r_{j}-r_{i}\right) w\left(\left|r_{j}-r_{i}\right|\right)\right]$

where $d$ is the number of space dimensions and $N$ is the total number of particles in the domain. This model is not sensitive to absolute pressure. This is consistent with the property of incompressible fluids, which only depends on the relative pressure distribution [11]. Thus equation can be rearranged as follows:

$\nabla \phi_{i}=\frac{d}{n^{0}} \sum_{j \neq i}^{N}\left[\frac{\phi_{j}-\phi_{i}^{\prime}}{\left|r_{j}-r_{i}\right|^{2}}\left(r_{j}-r_{i}\right) w\left(\left|r_{j}-r_{i}\right|\right)\right]$

where any constant value is allowed to $\phi^{\prime}{ }_{i}=\min \left(\phi_{j}\right)$ for any $j$ satisfying $w\left(\left|r_{j}-r_{i}\right|\right) \neq 0$.

A time-dependent diffusion problem with respect to $\phi$ is represented by Laplacian as:

$\frac{d \phi}{d t}=\nu \nabla^{2} \phi$

where $v$ is the diffusion coefficient. The variance of distribution of $\phi$ increases by $2 d v \Delta t$ during time step $\Delta t$, where $d$ is the number of space dimensions. In the current model, part of quantity $\phi_{i}$ of particle $i$ is distributed to the neighboring particles according to the kernel function such that the variance increase is equal to $2 d v \Delta t$. Thus, the quantity transferred from particle $i$ to $j$ is:

$\Delta \phi_{i \rightarrow j}=\frac{2 d v \Delta t}{n^{0} \lambda} \phi_{i} \theta w\left(\left|r_{j}-r_{j}\right|\right)$

where

$\lambda=\frac{\int_{V} w(r) r^{2} d V}{\int_{V} w(r) d V}$

$\lambda$ is a parameter by which the variance is equal to that of the analytical solution. The Laplacian operator is formulated as:

$\nabla^{2} \phi_{i}=\frac{2 d}{n^{0} \lambda} \sum_{j \neq i}\left(\phi_{j}-\phi_{i}\right) w\left(\left|r_{j}-r_{i}\right|\right)$

The Laplacian operator is conservative since the quantity lost by particle $i$ is just obtained by particles $j$.

\subsection{Finite Volume Method}

The finite volume method (FVM) is a discretization technique for partial differential equations and uses a volume integral formulation of the problem with a finite partitioning set of volumes to discretize the equations. This method has several applications in the area of fluid mechanics $[12,13]$. The three-dimensional incompressible and isothermal flow is mathematically described by the conservation laws, i.e., the 
conservation of mass and conservation of momentum [13]. These governing equations (1) and (2) can be cast into the following equations [13]:

$\nabla \cdot u=0$

$\frac{\partial u}{\partial t}+u \cdot \nabla u=-\frac{1}{\rho} \nabla p+g+v \nabla^{2} u$

In the FVM, the differential equations are written in an integral form for each volume. Boundary conditions are also taken into account for the volumes in the vicinity of the boundaries. A set of discretized equations is obtained and solved to obtain velocity and pressure fields.

The FVM implementation on Ansys Fluent was used in this work. The velocity-pressure equations were solved by the PISO algorithm [14]. The QUICK scheme [15] was used for the discretization of the momentum equations and the PRESTO! scheme was chosen for pressure discretization.

\section{NUMERICAL PROCEDURE}

A numerical study was conducted in order to compare the MPS method with the FVM. The laminar flow of a Newtonian fluid in a microchannel with a narrow constriction was used as a case study for this comparison. The fluid used for both numerical methods was water with $1000 \mathrm{~kg} / \mathrm{m}^{3}$ density and $1.0 \times 10^{-3}$ Pa.s viscosity. Fig. (1) shows the geometry used. The inlet and outlet regions have $30 \mu \mathrm{m}$ of width and $30 \mu \mathrm{m}$ of length. The narrow channel has $11.5 \mu \mathrm{m}$ of width and $100 \mu \mathrm{m}$ of length. The channel has a depth of 11.5 $\mu \mathrm{m}$.
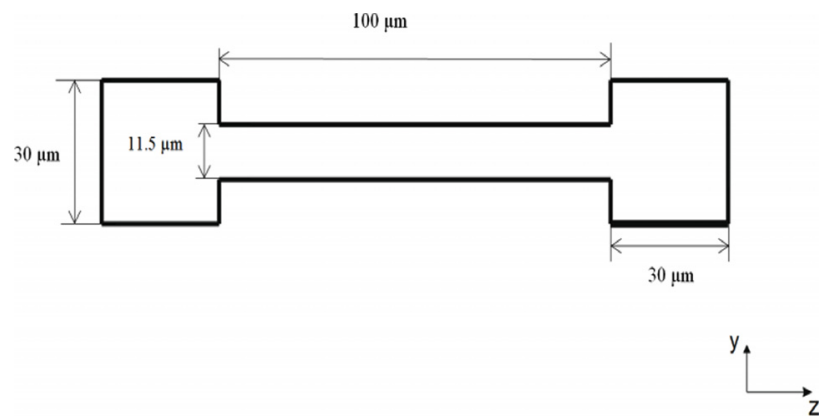

Fig. (1). Schematic representation of the microchannel.

Fig. (2) shows the computational mesh generated in $\mathrm{An}$ sys Meshing 13.0. A mesh with 36000 predominantly hexahedral elements was used in all Ansys Fluent simulations.

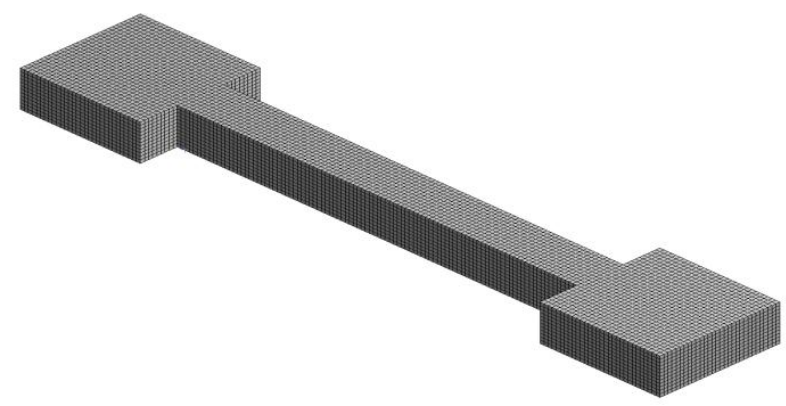

Fig. (2). Computational mesh (FVM).
To simulate the flow by the MPS method, it is necessary to select a small domain representing the problem under study. Hence, a section of the microchannel with $30 \mu \mathrm{m}$ length was selected (see Fig. 3). A periodic domain is used assuring the behaviour of a channel with an infinite length.

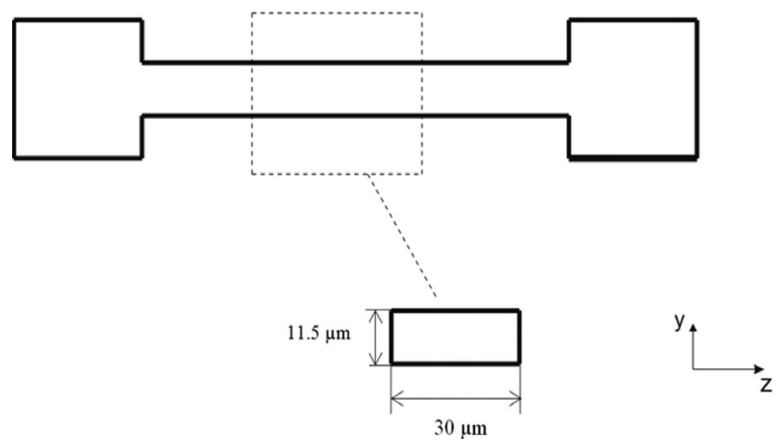

Fig. (3). Section of the channel used to perform the MPS method.

The MPS method discretizes the fluid contained in the domain in different particles. The accuracy of the solution that can be obtained by the MPS method depends on the initial particle distance $\left(r_{0}\right)$. There is no consensus regarding this parameter as several research works have used different values for the initial particle distance $[3,6,16,17]$. The value used must be a compromise between the accuracy of the domain discretization and the calculation time. A lower value of $r_{0}$ would increase discretization accuracy but would also increase the calculation time. To understand the effect of the initial particle distance on the accuracy of the numerical solution two particle distances were studied, $r_{0}=0.41$ $\mu \mathrm{m}$ and $r=0.205 \mu \mathrm{m}$. Fig. (4) shows a 3D representation of the initial particle distribution in the domain for $r_{0}=0.41$ $\mu \mathrm{m}$.

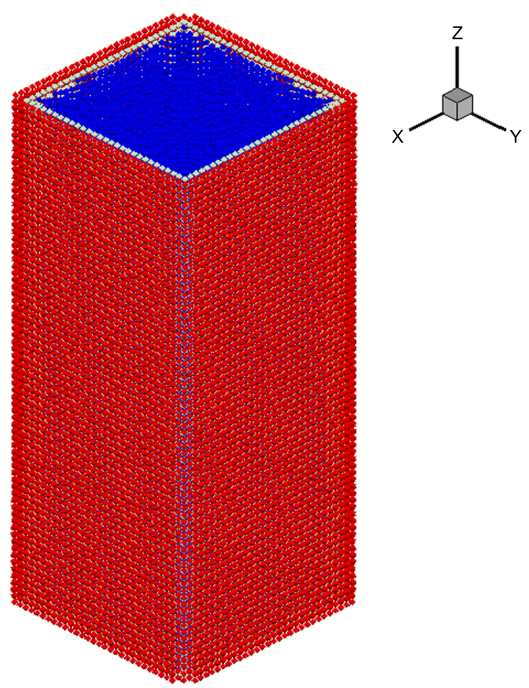

Fig. (4). 3D model of MPS method.

In Fig. (5), it is possible to observe different colors corresponding to different particle locations, i.e. the particles have colors corresponding to their type. Thus, particles having a dark blue color correspond to the fluid, the particles colored by green correspond to the channel wall and red particles correspond to the "ghost particles" essential to implement the boundary conditions. 


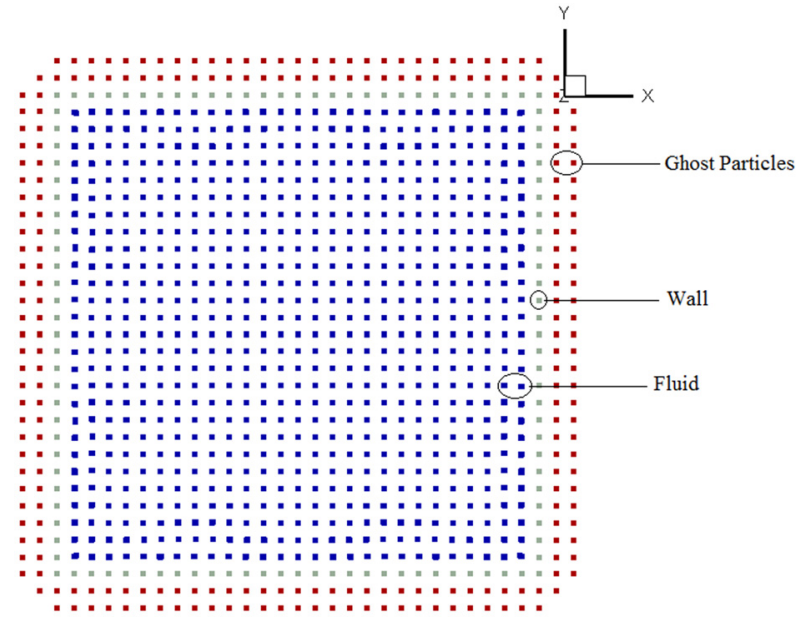

Fig. (5). Particles corresponding to different particle locations: dark blue particles correspond to the fluid, green particles correspond to the channel wall and red particles correspond to the ghost particles to implement the boundary conditions.

It is worth mentioning that in simulation carried out by Ansys, the no-slip boundary condition was used for the walls, the inlet velocity was set to $0.0023 \mathrm{~m} / \mathrm{s}(\mathrm{Re}=0.047)$ or 0.0115 $\mathrm{m} / \mathrm{s}(\mathrm{Re}=0.237)$ and the outlet pressure was set to zero. For the particular case of the MPS method, the no-slip boundary condition was also specified for the walls and the pressure drop calculated by the Ansys simulations was imposed between the outlet and the inlet.

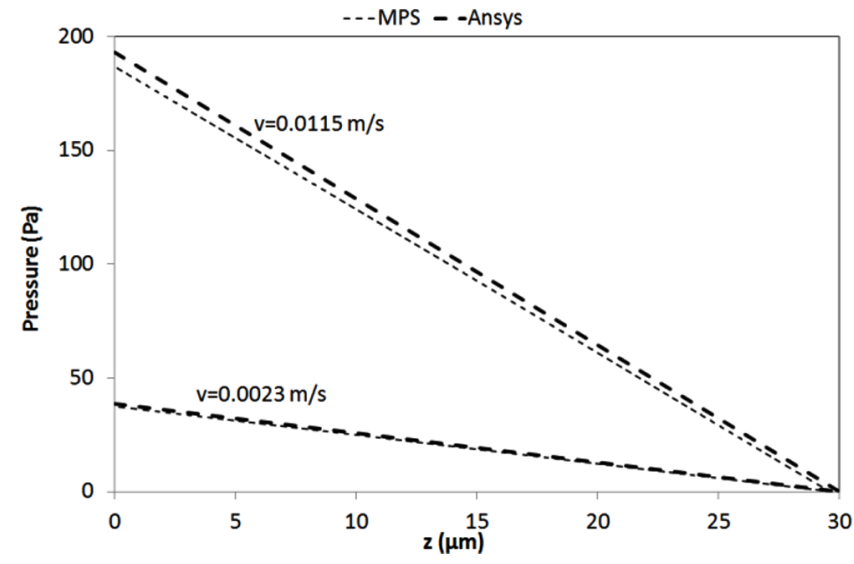

Fig. (6). Pressure along a section of the microchannel obtained by the two different numerical methods $\left(r_{0}=0.41 \mu \mathrm{m}\right.$ was used in the MPS method).

\section{RESULTS AND DISCUSSION}

Two numerical simulations were performed in Ansys Fluent, with inlet velocities of $0.0023 \mathrm{~m} / \mathrm{s}$ and $0.0115 \mathrm{~m} / \mathrm{s}$, respectively. The results were used to calculate the pressure drop along the channel.

For the inlet velocity of $0.0023 \mathrm{~m} / \mathrm{s}$, a pressure drop of $1.25821 \mathrm{~Pa} / \mu \mathrm{m}$ was obtained along the channel, while for the velocity of $0.0115 \mathrm{~m} / \mathrm{s}$ the pressure drop was $6.29139 \mathrm{~Pa} / \mu \mathrm{m}$.

The pressure drop obtained by Ansys was used in the MPS simulations. Note that, there is a small difference be- tween MPS and Ansys pressure drops. This difference was mainly due to a small error in the MPS domain boundary limit.

Fig. (7) shows the velocity contours obtained by the two methods under study. Qualitatively, both methods have the same behavior, i.e., they exhibit a maximum velocity in the center of channel while in the region near the walls the velocity is zero.

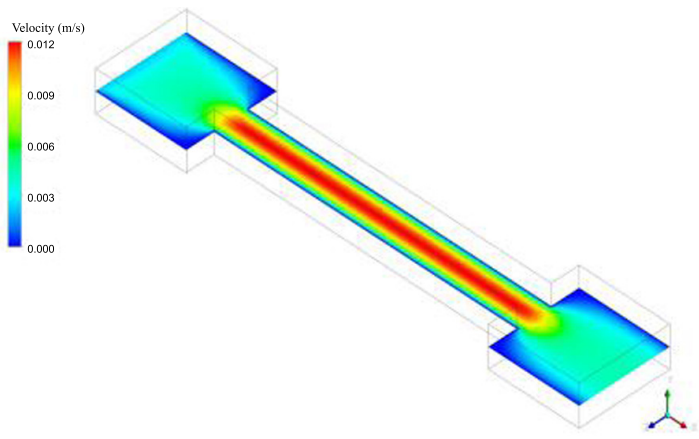

(a)

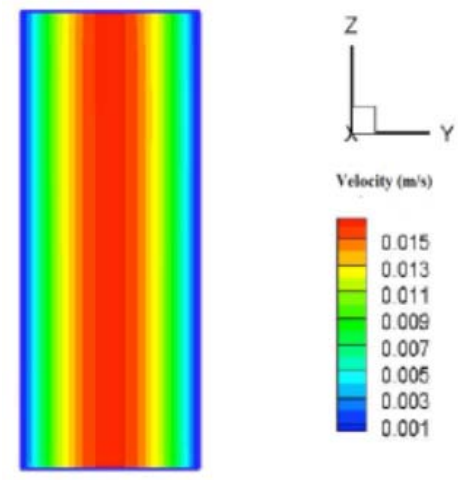

(b)

Fig. (7). Visualization of the numerical flow with pressure drop = $1.25821 \mathrm{~Pa} / \mu \mathrm{m}$ obtained by a) Ansys and b) MPS.

The velocity profiles obtained by both methods and the profile obtained for the theoretical model of Poiseuille flow in a rectangular channel [18] are represented in Fig. (8). The Poiseuille for a rectangular channel equation is given by:

$v_{z}(x, y)=\frac{4 h^{2} \Delta p}{\pi^{3} \eta L} \sum_{n, o d d}^{\infty} \frac{1}{n^{3}}\left[1-\frac{\cosh \left(n \pi \frac{y}{h}\right)}{\cosh \left(n \pi \frac{w}{2 h}\right)}\right] \sin \left(n \pi \frac{x}{h}\right)$

where $\eta$ is the fluid viscosity, $w$ is the channel width and $h$ is the channel depth.

All results were obtained in the midplane of the microchannel constriction. From Fig. (8), it can be seen that the velocity profile obtained by the FVM coincides with the velocity profile obtained by the Poiseuille model. The velocity profiles obtained by the MPS method are not independent of the particle distance and deviate from the theoretical profile. The deviation between the profile obtained by the MPS method and the theoretical profile is smaller for the smaller initial particle distance $\left(r_{0}=0.205 \mu \mathrm{m}\right)$, suggesting that the 


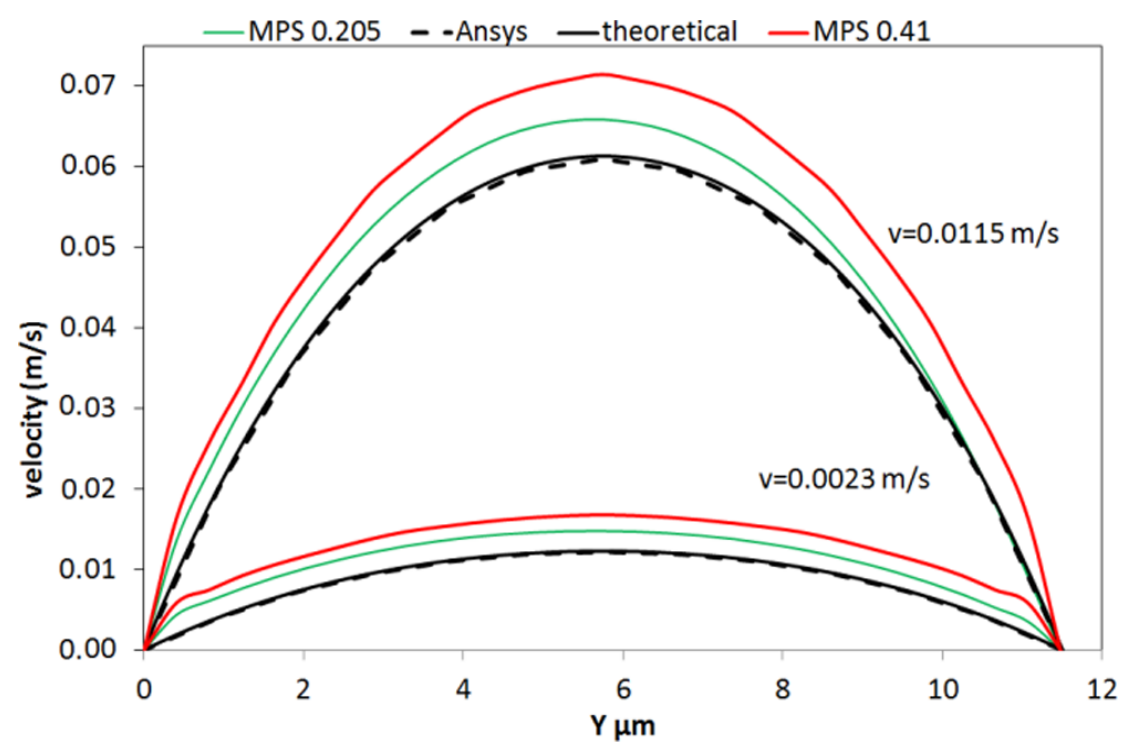

Fig. (8). Velocity profiles obtained by both numerical methods and theoretical model for the channel midplane.

solution obtained by the MPS method converges to the correct solution as the particle distance decreases. A particle distance smaller than $0.205 \mu \mathrm{m}$ is necessary to improve the accuracy of the solution obtained by the MPS method.

\section{CONCLUSION AND FUTURE DIRECTIONS}

The flow of a Newtonian fluid in a microchannel was solved by two methods, an FVM method and the MPS method. The results obtained by the MPS method depend on the particle distance, and even for the smallest particle distance studied, deviate from the exact theoretical solution and from the FVM. We believe that by reducing the particle spacing it is possible to improve the agreement between the two methods. Ongoing numerical simulations are currently being performed and results will be published in due time.

The current study is a first step to study in detail the motion and deformation of red blood cells flowing through narrow microchannels. The obtained results have shown the potential of the MPS to investigate the flow of single phase fluid in microfluidic systems. Further work is required to validate the implementation and to evaluate the method when applied to two-phase flows, such as blood flow.

\section{CONFLICT OF INTEREST}

The authors confirm that this article content has no conflict of interest.

\section{ACKNOWLEDGEMENTS}

The authors acknowledge the financial support provided by PTDC/SAU-ENB/116929/2010 and EXPL/EMSSIS/2215/2013 from FCT (Science and Technology Foundation), COMPETE, QREN and European Union (FEDER). D. Bento acknowledge the financial support provided by SFRH/BD/91192/2012 from FCT (Science and Technology Foundation), COMPETE, QREN and European Union (FEDER). The authors are also very grateful to Dr. Alberto
Gambaruto (Bristol University) for helpful explanations and suggestions regarding the MPS method.

\section{REFERENCES}

[1] Lima, R.; Ishikawa, T.; Imai, Y.; Yamaguchi, T. Blood flow behavior in microchannels: advances and future trends. In: Single and two-phase flows on chemical and biomedical engineering. Ricardo DM, Rui L, Antonio A, Teresa MM, Eds.; Bentham Sci., 2012. p. 513-547.

[2] Wang, T.; Xing, Z. Characterization of Blood Flow in Capillaries by Numerical Simulation. J. Mod. Phy., 2010, 1, 349-356.

[3] Tsubota, K.; Wada, S.; Yamaguchi T. Particle method for computer simulation of red blood cell motion in blood flow. Comput. Methods Programs Biomed., 2006, 83, 139-146.

[4] Miyazaki, H.; Yamaguchi, T. Formation and destruction of primary Thrombi under the influence of blood and von willebrand factor analyzed by a discrete element method. Biorheology, 2003, 40, 265-272.

[5] Tsubota, K.; Wada, S.; Kamada, H.; et al. A Particle Method for Blood Flow Simulation, -Application to Flowing Red Blood Cells and Platelets. J. Earth Simulator, 2006, 5, 2- 7.

[6] Imai, Y.; Kondo, H.; Ishikawa, T.; Teck Lim, C.; Yamaguchi, T. Modeling of hemodynamics arising from malaria infection. J. Biomech., 2010, 43, 1386-1393.

[7] Kamada H, Tsubota K, Nakamura M, Wada, S.; Ishikawa, T.; Yamaguchi, T. A three-dimensional particle simulation of the formation and collapse of a primary thrombus. Int. J. Numer. Method Biomed. Eng., 2010, 26, 488-500.

[8] Koshizuca, S.; Oka, Y. Moving-Particle Semi-Implicit Method for Fragmentation of Incompressible Fluid. Nucl. Sci. Eng., 1996, 123, 421-343.

[9] Ahmadian, M.T.; Firoozbakhsh, K.; Hasanian, M. Simulation of red blood cell motion in microvessels using modified moving particle semi-implicit method. Scientia Iranica, 2012, 19, 113-118.

[10] Khayyer, A.; Gotoh, H. Modified Moving Particle Semi-implicit methods for the prediction of 2D wave impact pressure. Coast. Eng., 2009, 56, 419-440.

[11] Yoon, H.Y.; Koshizuka, S.; Oka, S. A Particle-Gridless Hybrid Method for Incompressible Flows. Int. J. Numer. Meth. Fluids, 1999, 30, 407-424.

[12] Chang, R.; Yang, W. Numerical simulation of mold filling in injection modeling using a three-dimensional finite volume approach. Int. J. Numer. Meth. Fluids, 2001, 37, 125-148.

[13] Lan, C.W.; Liu, C.C.; Hsu, C.M. An Adaptive Finite Volume Method for Incompressible Heat Flow Problems in Solidification. J. Comput. Phys., 2002, 178, 464-497. 
[14] Issa, R.I. Solution of the implicitly discretised fluid flow equations by operator-splitting. J. Comput. Phys., 1986, 62, 40-65.

[15] Leonard, B.P. Order of accuracy of QUICK and related convectiondiffusion schemes. Appl. Math. Model., 1995, 19, 640-653.

[16] Tsubota, K.; Wada, S. Elastic force of red blood cell membrane during tank-treading motion: Consideration of the membrane's natural state. Int. J. Mech. Sci., 2010, 52, 356-364.

Received: July 03, 2015
Revised: September 14, $2015 \quad$ Accepted: November 23, 2015
[17]

Alizadehrad, D.; Imai, Y.; Nakaaki, K.; Ishikawa, T.; Yamaguchi, T. Quantification of red blood cell deformation at high-hematocrit blood flow in microvessels. J. Biomech., 2012, 45, 2684-2689.

[18] Bruus, H. Theoretical microfluidics. third edition ed. Department of Micro and Nanotechnology Technical University of Denmark; 2006 